\title{
PARASITISMO INTESTINAL Y ABSORCIÓN DE ANTICONVULSIVANTES
}

Jorge Apolinar Eslava Cobos MD*, Ignacio Salgado Castañeda MD**, Ramón Antonio Quintero Almenárez $\mathrm{MD}$,***

\section{Resumen}

Es un estudio realizado para determinar si existe algún tipo de interacción entre el parasitismo intestinal y la absorción de los medicamentos anticonvulsivantes (AC). Se incluyeron los pacientes que recibían AC (fenitoína, carbamazepina o ácido valproico) y que además tenían parasitismo intestinal documentado a través de exámenes coprológicos seriados por concentración. A estos enfermos se les midió la variación intraindividual de los niveles de AC antes y después de la desparasitación específica según el microorganismo. Participaron diez pacientes parasitados con diferentes microorganismos (Entamoeba histolitica, Blastocystis hominis y Endolimax nana) que al ser desparasitados el $60 \%$ de ellos aumentaron los niveles séricos de $\mathrm{AC}$, siendo este incremento igual para carbamazepina (66\%) y fenitoína $(66 \%)$.

Palabras clave: anticonvulsivantes, absorción, parásitos intestinales, niveles séricos, fenitoína, carbamazepina, ácido valproico.

Abreviaturas: AC, anticonvulsivantes; PI, parásitos intestinales.

\section{Introducción}

El conocimiento cada vez más profundo de los diversos fenómenos farmacológicos relacionados con el uso de los AC como es la absorción, se ha convertido en una de las herramientas importantes en el control de la epilepsia.

El presente estudio se realiza partiendo de la hipótesis fisiopatológica que los PI tienen la posibilidad biológica de interferir en la absorción de medicamentos. Conocemos de antemano dos estudios publica$\operatorname{dos}^{1,2}$ en los cuales los autores por encontrar cambios en los niveles séricos de fármacos $\mathrm{AC}$ luego de la desparasitación, sugieren la posibilidad de que los PI pueden interferir en la absorción adecuada de AC.

Fecha recibido: noviembre 15 de 2006 - Fecha aceptado: febrero 22 de 2007

* Neurólogo, especialista en epilepsia y neurología infantil, Universidad del Rosario, Instituto Colombiano de Neurociencias. Ex -jefe del servicio de neurología del Hospital de San josé.

* Neurólogo, instructor de postgrado en neurología, Fundación Universitaria de Ciencias de la Salud, Hospital de San José, Servicio de neurología, Clínica del Country.

*** Residente de neurología, Fundación Universitaria de Ciencias de la Salud, Hospital de San José.
Los medicamentos estudiados fueron fenitoína, ácido valproico y carbamazepina, ampliamente conocidos y utilizados en el campo de la neurología y la psiquiatría. Según lo descrito en la literatura, ${ }^{3}$ los niveles séricos de AC pueden tener una variación cercana al $4.7 \%$ en un mismo individuo. Por esta razón consideramos como significativos los cambios por encima o por debajo de esta cifra.

Es de gran importancia el estudio de estos fenómenos en países en vía de desarrollo como el nuestro, en el cual el PI es endémico y no siempre es posible medir las concentraciones séricas de AC.

\section{Métodos}

Participaron en el estudio los primeros diez pacientes con diagnóstico clínico de epilepsia del servicio de neurología, de ambos sexos, mayores de dos años de edad y que reunieron las siguientes exigencias:

- Tener un estado estable en la concentración sérica 
de fenitoína, carbamazepina y ácido valproico, definido por el tiempo de tratamiento, esto es mayor a 15 días.

- PI definido como la presencia de Entamoeba histolitica, Blastocystis hominis o Endolimax nana en el examen coprológico por concentración. Todos los estudios se realizaron en el laboratorio del Hospital de San José con el mismo método de concentración de heces (técnica de Burrows).

- Tratamiento con tabletas de metronidazol genérico $500 \mathrm{mg}$ cada ocho horas por vía oral durante cinco días.

- Determinación de niveles séricos de fenitoína, carbamazepina y ácido valproico en el mismo laboratorio y con la misma normatividad técnica (inmunoensayo).

- Control de niveles séricos de AC entre cinco y quince días después de finalizado el tratamiento.

- Aprobación por el comité de ética institucional del Hospital de San José y la Fundación Universitaria de Ciencias de la Salud.

- Haber autorizado su participación en el estudio a través de un consentimiento informado.

- Se consideró variación importante una diferencia entre las dos mediciones de niveles de medicamentos que estuvieran por encima o por debajo de 4.7\% (Delta 4.7\%).

\section{Resultados}

Todos los diez pacientes eran procedentes de la región andina y de área urbana estratos uno a tres, correspondiendo el $60 \%$ a sexo masculino y $40 \%$ al femenino. La edad promedio fue de 51.2 años. De los pacientes que recibieron carbamazepina (tres), dos $(66 \%)$ presentaron incremento de niveles y uno (33\%) no presentó cambio.
De los seis con fenitoina cuatro (66\%) elevaron los niveles y en dos (33\%) los niveles de control fueron inferior a los cuantificados en predesparasitación. Ambos refirieron después tener poca adherencia al tratamiento anticonvulsivo. El paciente que recibía ácido valproico, presentó una disminución de los niveles postdesparasitación.

Todos los casos con incremento de los niveles séricos fueron mayores a 4.7\%, cifra establecida según la literatura, ${ }^{3}$ como máxima variación intraindividual normal. En la Tabla 1 se resumen los hallazgos.

\section{Discusilón}

Con frecuencia en la práctica clínica observamos pacientes que con dosis adecuadas de medicamentos AC no logran conseguir niveles séricos suficientes, lo que repercute en forma directa sobre el control de las crisis epilépticas. Se plantea la posibilidad de una mala farmacocinética relacionada con alteraciones en la absorción de la medicación, que puede alterarse por alimentos, otros medicamentos y factores potenciales como la presencia de parásitos que tapizan la mucosa y disminuyen así la superficie de absorción en el intestino. Se han descrito casos con Gardia lamblia y Entamoeba histolitica. ${ }^{1,2}$ Estos hallazgos no serían del todo inesperados ya que estos dos microorganismos son conocidos patógenos que causan síndromes de malabsorción, algo menos explicable es haber encontrado en nuestro estudio casos de pacientes con parásitos como Blastocystis hominis y Endolimax nana que son parásitos usualmente no patógenos salvo en situaciones especiales, ${ }^{4,5,6}$ en los cuales al tratarlos hubo incremento en los niveles séricos. Dos pacientes tuvieron descenso de los niveles de fenitoína, explicable por la mala adherencia e irregularidad al tratamiento que refirieron.

Uno que recibió ácido valproico también presentó descenso de los niveles en el control, tal vez esté relacionado con el hecho que buena parte de estos parásitos habitan en intestino delgado y la mayoría de la absorción de ácido valproico es en el intestino grueso. ${ }^{7}$ Otro hallazgo importante en nuestro trabajo 


\begin{tabular}{|c|c|c|c|c|c|c|c|c|}
\hline \multicolumn{9}{|c|}{ Tabla I. Relación de los casos y las variables } \\
$\begin{array}{c}\text { Caso } \\
\text { número }\end{array}$ & Sexo & $\begin{array}{c}\text { Edad } \\
(\text { años })\end{array}$ & Coprológico & Medicamento & $\begin{array}{c}\text { Nivel } \\
\text { sérico } \\
(\text { Mcg/ } \\
\text { ml) }\end{array}$ & Desparasitación & $\begin{array}{c}\text { Nivel sérico } \\
\text { posterior } \\
(\text { Mcg/ml) }\end{array}$ & Delta \\
\hline 1 & masculino & 26 & $\begin{array}{c}\text { Blastocystis } \\
\text { hominis }\end{array}$ & Fenitoina & 17 & Metronidazol & 21 & $17 \%+$ \\
\hline 2 & masculino & 54 & $\begin{array}{c}\text { Entamoeba } \\
\text { histolitica }\end{array}$ & Fenitoina & 6 & Metronidazol & 3.6 & $\mathbf{4 0 \% =}$ \\
\hline 3 & masculino & 68 & $\begin{array}{c}\text { Blastocystis } \\
\text { hominis }\end{array}$ & Fenitoina & 11 & Metronidazol & 13 & $18 \%+$ \\
\hline 4 & femenino & 43 & $\begin{array}{c}\text { Blastocystis } \\
\text { hominis }\end{array}$ & Carbamazepina & 9.7 & Metronidazol & 11.1 & $\mathbf{1 4 \% +}$ \\
\hline 5 & femenino & 33 & $\begin{array}{c}\text { Blastocystis } \\
\text { hominis }\end{array}$ & Ácido valproico & 121 & Metronidazol & 83 & $\mathbf{3 1 \% =}$ \\
\hline 6 & masculino & 37 & $\begin{array}{c}\text { Entamoeba } \\
\text { histolitica }\end{array}$ & Fenitoina & 3.4 & Metronidazol & 5 & $\mathbf{4 7 \% +}$ \\
\hline 7 & masculino & 68 & $\begin{array}{c}\text { Entamoeba } \\
\text { histolitica }\end{array}$ & Fenitoina & 19 & Metronidazol & 3.4 & $\mathbf{1 7 \% -}$ \\
\hline 8 & masculino & 72 & $\begin{array}{c}\text { Endolimax } \\
\text { nana }\end{array}$ & Fenitoina & 15.5 & Metronidazol & 28.2 & $\mathbf{8 1 \% +}$ \\
\hline 9 & masculino & 74 & $\begin{array}{c}\text { Endolimax } \\
\text { nana }\end{array}$ & Carbamazepina & 3 & Metronidazol & 3 & $\mathbf{0} \%$ \\
\hline 10 & femenino & 52 & $\begin{array}{c}\text { Endolimax } \\
\text { nana }\end{array}$ & Carbamazepina & 6.7 & Metronidazol & 10.6 & $\mathbf{5 8 \% +}$ \\
\hline
\end{tabular}

$\mathrm{Mcg} / \mathrm{ml}=$ microgramos por mililitro.

$\%+=$ Porcentaje que aumentaron los niveles post-desparasitación.

$\%=\quad$ Porcentaje que disminuyeron los niveles post-desparasitación.

es haber encontrado parásitos, aunque en baja cifras, en una ciudad como Bogotá con agua potable y adecuadas condiciones sanitarias, que debería ser menos que la prevalencia en Colombia. ${ }^{6}$

\section{Conclusiones}

- $\quad$ E1 PI, incluyendo aquellos que producen pocas manifestaciones clínicas, puede interferir con la absorción de los AC en el sistema digestivo.

- La interferencia parece ser similar para fenitoína y carbamazepina, mas no para el ácido valproico en el cual no encontramos cambios.
- Es necesario explorar más a fondo la posible interferencia de los AC en el sistema gastrointestinal.

- Se hace necesario realizar estudios actualizados para conocer la verdadera prevalencia de PI en áreas urbanas grandes como es el caso de Bogotá.

\section{Referenclas}

1. Vega G.Efectos de la giardiasis sobre la absorción de fenitoína. .En:Trabajos libres VI reunión anual Academia Mexicana de Neurología, noviembre de 1982,Morelia,Mich. México.

2. Eslava-Cobos J.¿Influye el parasitismo intestinal sobre la absorción de anticonvulsivantes ?Univ.Med.Vol.26 Numero 4.1984:261-262. 
3. Burt Martha,Anderson D,Kloss Julie.Evidence-based implementation of free phenytoin therapeutic drug monitoring.Clinical Chemistry 46:8.2000 p 1132-1135.

4. Doyle.P.Journal of clinical microbiology,Jan 1990 p $116-$ 121.

5. Hussain Qadri. journal of clinical microbiology,Nov 1989 p 2407-2409.
6. Botero D,Restrepo M.Parasitosis humanas $20034^{\circ}$ ed: 63-70.

7. Cato A 3rd, Pollack GM, Brouwer KL. Age-dependent intestinal absorption of valproic acid in the rat. Pharm Res.1995 Feb;12(2):284-90.
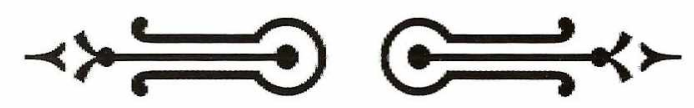

\section{(3)

Facultad de Instrumentación Quirúrgica

Código ICFES 270246100281100111100

Título otorgado: Intrumentador quirúgico.

Duración 8 semestres

No. créditos a cursar: 165.

Modalidad: presencial.
Oficina de admisiones: Carrera 19 No. 8 A-32 Edificio Docente, 1er. piso PBX. 3538100 ext. 3615

E-mail: instrumentacion@fucsalud.edu.co hppt: www.fucsalud.edu.co

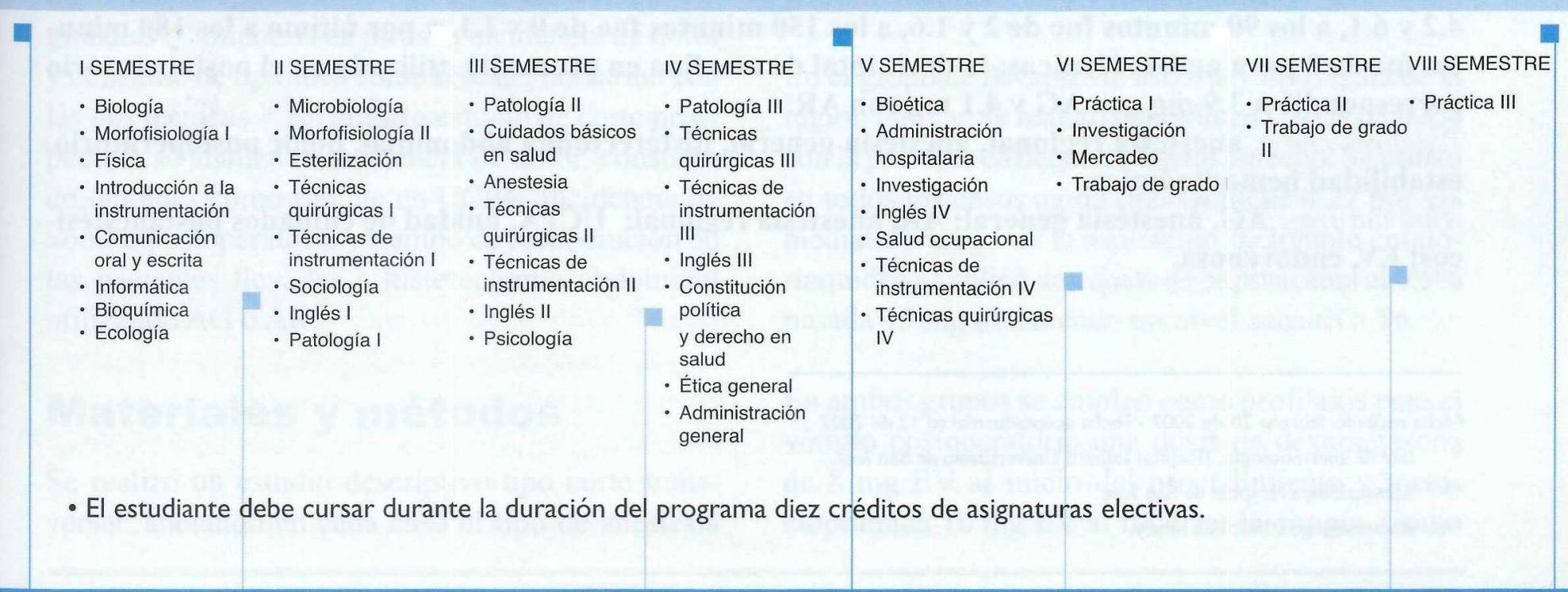

